\title{
Observation on the Nursing Effect of Prone Position Ventilation Applied to Children with Respiratory Failure
}

\author{
Aili Peng*, Litao Guo, Jing Xu, Jingrong Fan \\ Department of Critical Care Medicine, Children's Hospital Affiliated to Capital Institute of Pediatrics, Beijing 100020, China \\ Email:604916729@qq.com
}

\begin{abstract}
Objective - To compare the effects of two prone position ventilation methods on children with respiratory failure, to obtain a safer and more effective way to guide clinical nursing work. Methods - 48 cases of children who were admitted to the intensive care unit of our hospital from February 2018 to August 2019 and applied mechanical ventilation were divided into groups based on a random number table. The odd numbers were included in the experimental group (continuous prone position ventilation group, the duration of continuous prone position exceeded 12 hours, a total of 25 cases). The even numbers were included in the control group (intermittent prone position ventilation group, prone position for 3 hours at a time, alternating with supine position and lateral position, total prone position duration 12 hours, a total of 23 cases). Oxygenation index (OI), $\mathrm{PH}$ value, arterial partial pressure of carbon dioxide $\left(\mathrm{PCO}_{2}\right)$, arterial partial pressure of oxygen $\left(\mathrm{PO}_{2}\right)$ at $24 \mathrm{~h}, 48 \mathrm{~h}, 72 \mathrm{~h}$ of the two groups of children, as well as their ventilator use time and ICU hospital stay were compared. Results - Continuous prone position ventilation and intermittent prone position ventilation have no statistical significance on arterial blood carbon dioxide partial pressure $\left(\mathrm{PaCO}_{2}\right)$, arterial blood oxygen partial pressure $\left(\mathrm{PaO}_{2}\right)$, ventilator duration, ICU length of stay in children with respiratory failure $(\mathrm{P}>0.05)$, but with the increase of the total length of the prone position, when reaching more than 36 hours, the trend of oxygenation index (OI) of the experimental group and the control group can be seen to decline. Conclusion - In this study, by comparing the effects of two prone position ventilation modes, it was found that intermittent prone position ventilation and continuous prone position ventilation had no difference in the treatment of children with respiratory failure. When children are treated in continuous prone and intermittent prone positions, the total prone position can last up to 36 hours, which can effectively improve the clinical treatment effect. In view of the characteristics of pediatric patients and the difficulty in nursing critically ill patients with tracheal intubation, it is recommended that pediatric patients can use intermittent prone ventilation to complete treatment when the cumulative time in the prone position reaches more than 36 hours.
\end{abstract}

Keywords: prone position ventilation, respiratory failure, prone duration

Prone position ventilation (PPV) refers to placing the child in a prone position during mechanical ventilation. Recent studies have shown ${ }^{[1]}$ that the application of prone ventilation to children with respiratory failure can promote dorsal alveolar expansion and improve dorsal lung ventilation, thereby improving oxygenation, shortening the ventilator use time, and improving the prognosis. Many articles report ${ }^{[2]}$, for the adjuvant treatment of children's respiratory failure, prone position ventilation can promote symptom improvement, reduce the patient's respiratory power consumption and inhaled oxygen concentration, and have little impact on hemodynamics. It is easy to operate, non-invasive, and unnecessary. The advantages of special equipment and economy are becoming more and more widely used in clinical practice. The latest experiments show that ${ }^{[1]}$, the longer the prone position, the less lung damage. At present, there is no research data on the best time and method of prone position ventilation in the clinical treatment of respiratory failure in children. Long-term prone position ventilation leads to an increased risk of disengagement and pressure sores in children. The comparative data and nursing experience of the two types of prone position ventilation patients in the undergraduate room are reported as follows.

\section{Materials and methods}

\subsection{General information}

48 children with respiratory failure who were treated with mechanical ventilation in the intensive care unit of our hospital from February 2018 to August 2019 were selected.

Inclusion criteria are as follows. (1) In line with the guidelines for diagnosis and treatment of acute respiratory failure, type II respiratory failure: hypoxia with carbon dioxide retention $\left(\mathrm{PaO}_{2}<60 \mathrm{mmHg}, \mathrm{PaCO}_{2}>50 \mathrm{mmHg}\right)$, and the use of 
invasive ventilator therapy ${ }^{[3]}$. (2) Parents or guardians are informed and consent. (3) The doctor has a clear medical order.

Exclusion criteria are as follows. (1) Children with unstable hemodynamics: such as acute bleeding disorders; children with ECMO and continuous blood purification. (2) Increased intracranial pressure, neurosurgery. (3) Severe thoracic deformity, massive pneumothorax, thoracic and abdominal surgery. (4) Children who cannot tolerate prone position and rapid deterioration of vital signs during prone position ventilation.

A total of 48 children who met the inclusion criteria were grouped by a random number table, odd numbers were included in the experimental group (long-term prone position ventilation group, 25 cases in total), and even numbers were included in the control group (intermittent prone position group, repeated prone position ventilation methods, a total of 23 cases). See Table 1 for details.

Table 1. Comparison of general information of the two groups of children

\begin{tabular}{ccccc}
\hline Item & Number of cases [cases (\%)] & Gender [Male, (\%)] & Age [M(IQR), month] & $\begin{array}{c}\text { Critical case score } \\
\text { PCIS[M(IQR), points] }\end{array}$ \\
\hline Experimental group & $25(52.08 \%)$ & $16(64.00 \%)$ & $6.00(2.5,20.5)$ & $73.44 \pm 9.70$ \\
Control group & $23(47.92 \%)$ & $14(60.87 \%)$ & $4.00(2.00,10.00)$ & $76.00 \pm 8.14$ \\
Statistical value & - & - & -1.150 & -.986 \\
P & - & .823 & .250 & .309 \\
\hline
\end{tabular}

\subsection{Methods}

\subsubsection{Application of the ventilator}

Both groups of children were treated by oral tracheal intubation connected to an invasive ventilator. Servo-i universal ventilator from Sweden MAQUET company is used. Mechanical ventilation modes: regular frequency [pressure regulated volume control (PRVC), pressure control, intermittent positive pressure ventilation (IPPV), synchronized intermittent mandatory ventilation (SIMV) + pressure control, volume control] and high frequency (HFO). Adjust the parameter settings of the ventilator according to the lung conditions of the child, and observe the effect of ventilation after assisted ventilation. If there is no obvious human-machine confrontation, the tolerance is good, and the transcutaneous blood oxygen saturation $\left(\mathrm{SpO}_{2}\right)$ is maintained above $90 \%$, that is, the parameters are kept unchanged, and then the parameters are adjusted according to the blood gas results. When the child's blood gas results and ventilator parameters meet the conditions of weaning, weaning, and stop prone position after weaning.

\subsubsection{Prone position ventilation}

Twenty-five cases in the experimental group took the continuous prone position (long-term prone position ventilation). Accumulate 12 hours in the prone position; turn over due to operations during the prone position, and limit the time to turn over once in the prone position; concentrated operation and the operation time is limited to 20 minutes; the prone position can be turned over for more than 3 hours (continue to prone after the operation) Position); observe the cumulative time of 12 hours in the prone position during the day.

The 23 patients in the control group took the intermittent prone position (repetitive prone position ventilation). Prone position for 3 hours; supine or side-lying alternately; observe the prone position for a cumulative time of 12 hours.

\subsubsection{Nursing implementation}

The preparations for implementing prone position ventilation are as follows. Nursing evaluation: Comprehensive assessment of the child's state of consciousness, blood oxygen saturation, skin condition of the compressed area when lying prone, skin pressure ulcer score (a score lower than 16 points is a high-risk pressure ulcer, special attention should be paid to observe and do it in advance Good protection; keep the patient's respiratory tract unobstructed, suck sputum before operation; prepare for use: 2-4 soft pillows, 2-3 foam dressings. Prepare new electrodes.

During the implementation of the prone position, in order to improve the comfort of the child, give appropriate analgesic and sedative drugs according to the doctor's advice to reduce the fear of the child; use a closed suction tube to increase the secretion after the lungs are drained when lying prone; increase the number of sputum suction, reduce the number of disconnection of the ventilator, and maintain effective ventilator ventilation; strengthen inspections, and increase the frequency of observation when prone position for a long time. Observation content: the child's vital signs and state of consciousness; observe the condition of the compressed skin (face, shoulders, iliac, knees, ankles, etc.). Use an anti-decubitus air bed for the whole body, stick an AMP on the pressured part, massage the skin of the pressured part every 2 hours, put a soft pillow on the chest and abdomen, and observe the pipelines (whether they are unobstructed, the outer 
length of the tube, and the adhesion of the fixed tape); pay attention to teamwork every time your body position changes, and observe the tape fixed on each pipeline at any time to prevent loosening. Ensure the unobstructed pipeline, and prevent accidental falling off, suffocation, etc.

After the prone position is implemented, check the child's vital signs and consciousness, skin condition, and the condition of each pipeline. Ensure the unobstructed pipeline, prevent accidental falling off, suffocation, etc.

\subsubsection{Member training}

Prone position ventilation is difficult and dangerous. Therefore, it is necessary to formulate standardized procedures for uniform training of prone position performers and data collectors to avoid risks and adverse events and ensure the consistency of records. According to the "Prone Position Ventilation Operation Process" formulated by Tang Mengqin ${ }^{[4]}$ (including patient assessment, pre-operation preparation, operation steps, complications and precautions, and the end of prone position ventilation), standardize the prone position ventilation operation process for children .

\subsubsection{Data collection}

The $24 \mathrm{~h}, 48 \mathrm{~h}, 72 \mathrm{~h}$ related data of the experimental group and the control group are as follows. Oxygen index (Oxygen index, OI); the average of three daily blood gas tests: critical value of the human body $\mathrm{PH}$, partial pressure of oxygen $\left(\mathrm{PO}_{2}\right)$, partial pressure of carbon dioxide (Partial Pressure of Carbon Dioxide, $\mathrm{PCO}_{2}$ ); the duration of ventilator use and ICU stay in the experimental group and the control group.

\subsection{Statistical analysis}

SPSS 26.0 software was used for statistical analysis. The central tendency of non-normal distribution is expressed by the median (interquartile range) $[\mathrm{M}(\mathrm{IQR})]$, and the rank sum test (Mann-Whitney $\mathrm{U}$ test) is used; the central tendency of normal distribution or approximately normal distribution is expressed by the mean \pm The standard deviation (Mean $\pm \mathrm{SD}$ ) is represented by the chi-square $\left(\mathrm{x}^{2}\right)$ test for qualitative data and the independent sample $t$ test for quantitative data. The difference was statistically significant with $\mathrm{P}<0.05$.

\section{Results}

\subsection{Oxygenation status}

By comparing the oxygenation of the two groups of children, a trend was found: the OI of the control group and the experimental group decreased. However, the OI and blood oxygen partial pressure of the two groups were not statistically significant $(\mathrm{P}>0.05)$. See Table 2 .

Table 2. Comparison of oxygenation status of the two groups of children

\begin{tabular}{|c|c|c|c|c|c|c|}
\hline \multirow{2}{*}{ Item } & \multicolumn{3}{|c|}{ OI (mmHg) } & \multicolumn{3}{|c|}{$\mathrm{PO}_{2}(\mathrm{mmHg})$} \\
\hline & $24 \mathrm{hOI}$ & $48 \mathrm{hOI}$ & $72 \mathrm{hOI}$ & $24 \mathrm{hPO}_{2}$ & $48 \mathrm{hPO}_{2}$ & $72 \mathrm{hPO}_{2}$ \\
\hline Experimental group & $6.15(5.04,8.76)$ & $5.87(4.54,8.79)$ & $5.38(3.93,7.20)$ & $63.4(58.4,71.9)$ & $63.4(57.25,70.15)$ & $66.1(60.9,73.65)$ \\
\hline Control group & $5.92(4.29,6.98)$ & $4.75(3.91,6.65)$ & $4.70(3.07,6.19)$ & $65.8(59.3,73.3)$ & $64.0(59.2,70.2)$ & $65.1(60.1,79.8)$ \\
\hline Statistical value & -.815 & -1.610 & -.898 & -.650 & -.516 & -.361 \\
\hline $\mathrm{P}$ & .415 & .107 & .369 & .516 & .606 & .718 \\
\hline
\end{tabular}

\subsection{PH value and blood carbon dioxide partial pressure}

The comparison of $\mathrm{PH}$ value and blood carbon dioxide partial pressure between the continuous group and the discontinuous group was not statistically significant $(\mathrm{P}>0.05)$. See Table 3 .

Table 3. Blood gas PH and arterial carbon dioxide partial pressure of the two groups of children

\begin{tabular}{|c|c|c|c|c|c|c|}
\hline \multirow{2}{*}{ Item } & \multicolumn{3}{|c|}{ PH value } & \multicolumn{3}{|c|}{$\mathrm{PCO}_{2}(\mathrm{mmHg})$} \\
\hline & $24 \mathrm{hPH}$ & $48 \mathrm{hPH}$ & $72 \mathrm{hPH}$ & $24 \mathrm{hPCO}_{2}$ & $48 \mathrm{hPCO}_{2}$ & $72 \mathrm{hPCO}_{2}$ \\
\hline Experimental group & $7.38 \pm 0.051$ & $7.26 \pm 0.58$ & $7.40 \pm 0.047$ & $46(42.85,50.25)$ & $50.3(44.4,54.95)$ & $47.6(43.65,50.45)$ \\
\hline Control group & $7.39 \pm 0.053$ & $7.38 \pm 0.053$ & $7.40 \pm 0.040$ & $45.3(41.4,51.7)$ & $48.5(44.9,53.7)$ & $46.9(42.7,50.1)$ \\
\hline Statistical value & -.842 & -1.006 & -.533 & -.093 & -.691 & -.764 \\
\hline $\mathrm{P}$ & .873 & .096 & .613 & .926 & .489 & .445 \\
\hline
\end{tabular}




\subsection{Length of ventilator use, length of hospital stay and prognosis}

There was no significant difference between the two groups of ventilator use time and hospital stay $(\mathrm{P}>0.05)$. The 28 day prognosis of the experimental group and the control group showed that the death rate of the experimental group was $1 / 25(4 \%)$, and the death rate of the control group was $1 / 23(4.3 \%)$, and there was no statistical difference $(\mathrm{P}=.952)$. (Listed in Table 4)

Table 4. The duration of ventilator use, length of stay in ICU and prognosis of the two groups

\begin{tabular}{cccc}
\hline Item & Length of ventilator use (hours) & Length of stay in ICU (days) & Prognosis (death) $(\%)$ \\
\hline Experimental group & $156.00(122.50,258.00)$ & $14.00(10.50,18.50)$ & $1(4 \%)$ \\
Control group & $138.00(96.00,256.00)$ & $11.00(10.00,17.00)$ & $1(4.3 \%)$ \\
Statistical value & -.753 & -.649 &. \\
$\mathrm{P}$ & .451 & .516 & .952 \\
\hline
\end{tabular}

\section{Discussion}

Respiratory failure (respiratory failure, referred to as respiratory failure) is a serious disorder of lung ventilation and/ or ventilation caused by various reasons, so that effective gas exchange cannot be carried out, resulting in hypoxia with (or without) carbon dioxide retention, which causes a A series of clinical syndromes of physiological function and metabolic disorders. Chen $\mathrm{Xi}$ et al. pointed out ${ }^{[5]}$ that prone position ventilation can promote the recruitment of atrophic alveoli, increase the residual capacity of lung function, and improve lung oxygenation function. Compared with conventional mechanical ventilation in the supine position, ventilation in the prone position has obvious advantages and is considered to be a better mechanical ventilation position. Prone position ventilation therapy can promote gas exchange, reduce airway peak pressure and oxygen concentration under the premise of improving oxygenation function, and reduce lung damage and oxygen poisoning. It is an effective method for the treatment of respiratory failure ${ }^{[6]}$. The latest experiments show ${ }^{[1]}$ that the benefits of the prone position are related to the reduction of the harm of mechanical ventilation, so the longer the prone position, the less lung damage. A number of studies have pointed out ${ }^{[7]}$ that prone position ventilation can facilitate the discharge of secretions.

Paediatric patients are too young to live independently and cannot actively cooperate with treatment. When they are placed in a prone position or change their position for a long time, various catheters are susceptible to pressure and discounts, and it is difficult for nurses to observe. The secretions increase after the posture of the lungs, and the tape for fixing the tracheal intubation and the tape for the gastric tube are easily soaked, which increases the risk of dropping the tube and needs to increase the number of sputum suctions. Since the stratum corneum of children's skin is thinner than that of adults, and the epidermis and dermis are not closely connected ${ }^{[8]}$, when placed in the prone position for a long time, the risk of pressure injury increases, and it is necessary to increase the number of massages of the skin on the pressure site (1 time/2 hours). These factors lead to a significant increase in the difficulty of nursing and the workload of nursing.

In contrast, during intermittent prone position ventilation, due to frequent changes in body position, the child's local compression time is reduced, so the number of massages is significantly reduced. The training of operators involved in this project in the early stage is in place, and the operation is standardized. At present, the rate of decoupling and pressure injury of children in our department who are undergoing intermittent prone position ventilation are $0 \%$.

In this study, the two groups of children with long-term continuous prone ventilation and intermittent prone ventilation had no statistical significance in the length of ventilator use, length of hospital stay and prognosis $(\mathrm{P}>0.05)$. The oxygenation indexes of the two groups at 24 hours, 48 hours and 72 hours were $[6.15(5.04,8.76) 5.87(4.54,8.79)$ $5.38(3.93,7.20)][5.92(4.29,6.98) 4.75(3.91,6.65) 4.70(3.07,6.19) 65.8(59.3,73.3)]$. The oxygenation index at $72 \mathrm{~h}$ was lower than that at $48 \mathrm{~h}$ and $24 \mathrm{~h}$, indicating that both prone position ventilation methods can effectively improve oxygenation in children. The partial pressures of blood carbon dioxide at 24 hours, 48 hours, and 72 hours were [46(42.85,50.25) 50.3(44.4,54.95) 47.6(43.65,50.45)] [45.3(41.4,51.7) 48.5(44.9,53.7) $46.9(42.7,50.1)]$. The partial pressure of blood carbon dioxide at 72 hours in the prone position for a long time was lower than that at 48 hours, indicating that both prone position ventilation methods can improve carbon dioxide retention. It can be seen that, regardless of whether the prone position is continuous or intermittent, as long as the total length of the prone position reaches 36 hours or more, the two prone position ventilation methods used in the auxiliary treatment of ventilator are both effective and can be effectively improved for children with respiratory failure. Pulmonary ventilation and ventilation dysfunction. And the intermittent 
prone position has its irreplaceable advantages.

In summary, when pediatric patients are treated in the prone position and intermittent prone position, the total length of the prone position reaches 36 hours, which can improve the clinical treatment effect, and the choice of the two methods can make the nurse convenient for clinical care. Pediatric patients have the characteristics of poor cooperation, mobility, and skin sensitivity. Especially for critically intubated children, when prone for a long time, the increase of secretions increases the risk of decoupling and the difficulty of nursing. Combined with the research results, in order to avoid clinical nursing risks and reduce the difficulty of nursing, it is recommended that pediatric patients can use intermittent prone position ventilation to complete the treatment under the premise of ensuring that the cumulative time in the prone position reaches more than 36 hours.

\section{References}

[1] Luciano, Gattinoni, Paolo, et al. Prone position in acute respiratory distress syndrome. Rationale, indications, and limits. American Journal of Respiratory \& Critical Care Medicine. 2013; 188(11): 1286-1293.

[2] Fan Xuwei. Clinical observation of prone position mechanical ventilation adjuvant drug treatment of severe pneumonia in children. Modern Diagnosis and Treatment. 2017; 28 (1): 43-45.

[3] Pediatric Acute Lung Injury Consensus Conference Group. Pediatric acute respiratory distress syndrome: consensus recommendations from the Pediatric Acute Lung Injury Consensus Conference. Pediatric Critical Care Medicine. 2015; 16(5): 428-439.

[4] Tang Mengqin, the implementation and nursing of prone position ventilation in ICU. Psychologist. 2017; 23(24): 203204.

[5] Chen Xi, Wu Qiyun, Wang Xin, et al. Meta-analysis of the effects of prone position ventilation on patients with acute respiratory distress syndrome. Journal of Nursing of Chinese People's Liberation Army. 2016; 33(7): 20-23.

[6] Sud S, Friedrich JO, Adhikari NK, et al. Effect of prone positioning during mechanical ventilation on mortality among patients with acute respiratory distress syndrome: a systenatic review and meta-analysis. Canadian Medical Association Journal. 2014; 186(10): E381-E390.

[7] Lei Guangfeng, Zhang Xueqing, Zhang Suxia. Meta analysis of prone and supine ventilation in ALI/ARDS patients. Journal of Nursing Science. 2016; 31(22): 87-92.

[8] Chen Chen, $\mathrm{Lu}$ Wei, Wu Ling, etc. A systematic review of the literature on medical adhesive-related skin injuries. Journal of Nursing Science. 2016; 31(20): 99-103. 